\title{
Sentiment Analysis on Bengali Facebook Comments To Predict Fan's Emotions Towards a Celebrity
}

\author{
Md. Serajus Salekin Khan, Sanjida Reza Rafa, Al Ekram Hossain Abir, Amit Kumar Das* \\ Department of Computer Science and Engineering, East West University, Dhaka, Bangladesh
}

Received: April 29, 2021, Revised: July 12, 2021, Accepted: July 14, 2021, Available Online: July 23, 2021

\begin{abstract}
In this present era, sentiment analysis is considered one of the most rapidly growing fields of computer science study. It is a text mining technique that is automated and determines the emotion of a text. A text can be divided into many emotions using sentiment analysis. Since there are some studies on emotion analysis in the Bangla language, it is regarded as a key research area in the field of analyzing the Bangla language. This paper works with five different emotions: Happy, Sad, Angry, Surprised, and Excited. Apart from these emotions, our paper also deals with two categories, as Abusive and Religious. We used different machine learning techniques to train our dataset. We got an accuracy of $62 \%$ for Support Vector Machine (SVM), 58\% for Random Forest (RF), 55\% for K-Nearest Neighbors (KNN), 52\% for Naïve Bayes (NB), and 50\% for Neural Network.
\end{abstract}

Keywords: Sentiment, Celebrities, Machine Learning, tf-idf, svm, Emotion

This work is licensed under a Creative Commons Attribution-Non Commercial 4.0 International License.

\section{Introduction}

Sentiment analysis is also known as opinion mining, is the study of interpreting human feelings, which is the systematic detection, extraction, quantification, text interpretation, where NLP(Natural Language Processing) is used to investigate affective states and contextual intelligence. It is, in turn, a method for determining the emotional tone of a group of words, which is then used to get a better interpretation of the attitudes, opinions, and feelings expressed in an online mention.

Detecting underlying feelings has become a must in today's ever-changing world. We can gain major advantages in several fields by detecting the motive behind a human decision, which can range from buying a particular product to criminal motivation, employee-employer relationships, and so on. Sentiment analysis is a highly automated process that can be used to understand a viewpoint from spoken language, documents, or even video feeds.

Human Emotions are biochemical states resulting from neurophysiological shifts in the system [1]-[3] of the nerves that are linked to behavioral retaliation, feelings, thoughts. Psychologists have attempted to categorize the various kinds of feelings that people have. Few different ideas have arisen to categorize and describe the feelings that people feel. Psychologists have identified six basic emotions: sorrow, happiness, anxiety, disgust, rage, and surprise. Analysis of human reaction has exploded over the past two decades, with contributions from a variety of fields such as psychology, psychiatry, affective biology, sociology of feelings, computer science, medicine, history, and medicine. However, when researchers wanted to identify these basic human emotions from any text written by humans, speech, and facial expressions, the term 'Sentiment Analysis' is introduced in computer science.

The original academic studies of public opinion were performed during WWII and after WWII, with a mostly political impetus. However, contemporary sentiment research did not become mainstream until the mid-2000s and is mostly concentrated on online-based product ratings [4].
After the outbreak, the use of sentiment analysis has been extended to a number of other sectors, including stock market forecasting and terrorist attack reactions. Moreover, sentiment analysis is useful for social media monitoring because it helps us to see how the general public feels about a given subject. It has a wide range of applications and is highly efficient. Organizations all over the world are embracing the opportunity to derive information from social data.

However, when we went through with different research work on human emotion analysis, we found that there have been relatively few studies on emotion analysis in the Bangla language due to its language complexity, while several works have been done for English and other languages [5],[6]. Bangla is the world's fourth most common language, with about 250 million native speakers worldwide. As Bangla is one of the most popular languages and almost 250 million people are using Bangla to express their emotions, we emphasized Bangla to extract emotion. On the other hand, detecting any slang or abusive words in Bangla is comparatively more complex due to a lack of resources than in English [7],[8]. Moreover, there are religious variations among the Bengali speakers. People are using social media to defame different religious thoughts, and this is how religious violence is increasing day by day. Generally, people express their opinions and ideas through social media like Facebook, Twitter, etc., a considerable amount of data is available about various issues [9].

Being inspired by the application of sentiment analysis, in this paper, we proposed a method that will predict sentiment from text written in BANGLA using the SVM algorithm.

\section{Related Works}

Among various research areas of Computer science, sentiment analysis is considered one of the fastest-growing research topics. According to a review, the origins of sentiment analysis can be found in surveys on public opinion research at the turn of the twentieth century and text analysis conducted by a computational linguistics community in the 1990s [4]. 
Computer-based sentiment analysis moved towards the outbreak with the availability of texts on the web. This outbreak greatly impacted various fields by identifying the underlying emotion from any text or voice message. Many papers discussed the issue of sentiment analysis using a number of approaches. We had found several papers talking about our related field when we went through different papers.

Das. A. [10] et al. suggested a hybrid mechanism by integrating both rule-based and automated systems to derive the viewpoints from the text (Bangla and English). They have used Natural Language Processing (NLP) and SVM to implement this system. To get a better solution, Mahmudun M. et al. [9], used the concept of TF (term frequency) and IDF (inverse document frequency) values, and they extract the various characteristics of negative, positive, or neutral terms from text written in Bangla to get a more accurate result. Das D. [11] proposed an emotion monitoring method on a subject or event that used SentiWordNeT for both Bangla and English text and used sensebased affect scoring techniques. Hasan K.A. et al. [12] used SentiWordNet and WordNet to determine the polarity and meaning of words of the text to develop their methods for detecting sentiment from text written in the Bangla language. Chowdhury S. et al. [13] used the SVM and MaxEnt (Maximum Entropy) algorithm to extract sentiments from Bangla Microblog (Twitter) posts automatically, regardless of whether the polarity of the text is positive or negative. Go A. et al. [14] used three machine learning techniques: SVM, MaxEnt, and Naive Bayes to identify the emotions of Twitter messages with emoticons. Using HindiSentiWordNet (HSWN) and the Synset replacement algorithm, Pandey P. et al. [15] proposed a method for sentiment analysis of Hindi movie reviews. To extract emotion from any Bangla text, Tuhin R. A. et al. [16] proposed two machine learning techniques: the Naive Bayes Classification Algorithm and the Topical Method. The suggested methods have been used at both the article and sentence levels. Tembhurnikar S. D. et al. [17] developed a model based on LDA to analyze sentiment, which they used to rate tweets in terms of popularity. Umamaheswari K. et.al [18] used SVM and LDA to identify the viewpoint from the IMDB movie analysis dataset.

\section{Methodology}

Among the three broad areas of Machine learning, we used Supervised Learning. Since we worked on text classification and our dataset was multiclass labeled, supervised learning was the suitable approach for this kind of research. Because supervised classification of text is done when we have defined the classification categories [19]. This means that it requires the prelabeled dataset with correct values.

Again, machines do not understand free text, image, or video data. It can read only $1 \mathrm{~s}$ or $0 \mathrm{~s}$. To make data as machine-readable, the dataset must be transformed or encoded. For this case, we used Term Frequency-Inverse Document Frequency (TF-IDF) which is available in a python library called "Scikit-learn".

So, the framework of this study includes- cleaning dataset and transform it into vector form. Learn the transformed dataset in machine learning models. To learn our dataset in the machine, we used the Support Vector Machine (SVM) classifier in this research. SVM gives very outstanding results compared to other classifiers such as K-Nearest Neighbors, Naive Bayes, Random Forest, Neural Network, Random Forest, which were also used in this research to ensure the validity of the result. SVM is so famous for its kernel trick to handle nonlinear input shapes [20]. Generally, supervised learning-based sentiment analysis consists of two measures for document classification. As shown in Fig. 1, it learns a model by using the training data. The trained classifier evaluates the model's accuracy in the testing process by predicting the target class of unknown test data.

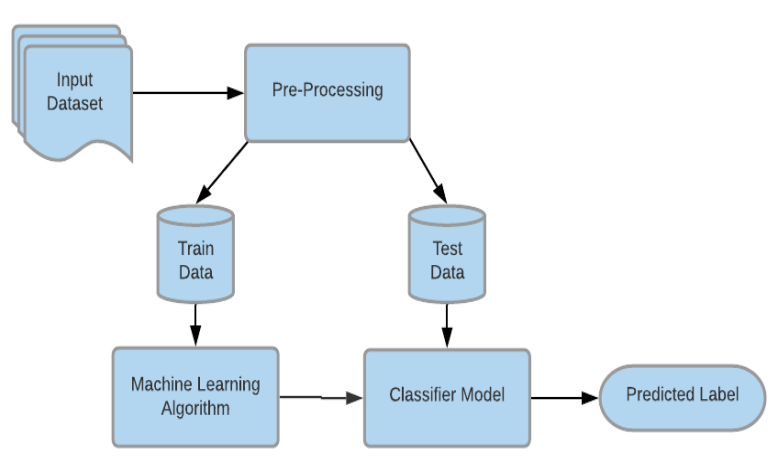

Fig. 1 Working Mechanism of proposed method

Table 1 Dataset Sample

\begin{tabular}{|c|c|}
\hline Comments & Emotion \\
\hline $\begin{array}{c}\text { সত্যি সাকিব ভাইয়া মন থেকে তোমাকে } \\
\text { অনেক ভালোবাসি }\end{array}$ & Happy \\
\hline $\begin{array}{c}\text { তোমার সেই গর্জন নিয়ে আবার মাঠে ফেরার } \\
\text { অপেক্ষায় আছি }\end{array}$ & Excited \\
\hline $\begin{array}{l}\text { তদের বড়লোকি জীবন দেখলে আমরা } \\
\text { ছোটলোকদের কষ্ট হয় }\end{array}$ & Sad \\
\hline একজন বিধর্মীর জন্য এত আবেগ ভালো না & Religious \\
\hline $\begin{array}{c}\text { জতো পারিস তেল লাগা লুচ্চার বাচ্চা লাব হবে } \\
\text { না }\end{array}$ & Abusive \\
\hline $\begin{array}{c}\text { গতি ১৪৫ কিঃমি বাংলার মিসাইল তাসকিনের } \\
\text { বিধ্বংসী বোলিংয়ে অবাক সবাই }\end{array}$ & Surprised \\
\hline $\begin{array}{c}\text { এখানেও এসে কিছু ব্যাক্তি উপদেশ দিতে শুরু } \\
\text { করবে যতসব নেকামি এই জাতি }\end{array}$ & Angry \\
\hline
\end{tabular}

\subsection{Data collection}

Data has been collected from some Facebook pages of Bangladeshi celebrities, including Cricketer, Actor, Actress, Youtuber, etc. For this research, data was specifically comments of general peoples on celebrity's Facebook pages. Facepager has been used for extracting comments from posts of some pages as it can fetch publicly available data from Facebook, YouTube, Twitter and other websites with JSON based API. To extract data with facepager, an access token is required. The token will be generated automatically when a profile is logged in via this tool. Another option to collect access token is a website called 'Graph API Explorer- Facebook Developer'. The Graph API allows us to read and write data to and from the Facebook social graph. Facepager takes an id of the desired pages or profiles which we want to use for extracting data. After extraction, it stores the raw data in a SQLite database and later those data can be exported as a csv excel sheet [9].

We have collected 63,000 comments as raw data, including Bangla, English, and a mix of both Bangla and English language called Banglish or other languages. For collection sources, we 
have used 12-15 pages of celebrities based on a 3-month duration. Table 1 shows some samples from our dataset, which represents a short scenario of the dataset.

\subsection{Data Cleaning}

Collected raw data has different varieties of comments where we required only pure Bangla text. It means that a text or comment should be written with only Bengali fonts and should not contain any other characters. Generally, in social media, people's comments in several types of language or mix of multiple language characters in a single comment. Since we need only Bangla comments, we had to clean and remove unnecessary data from the raw dataset as these data will create noise and reduce model accuracy.

The English and multilingual comments were removed first, leaving around 10,000 comments. The dataset after this process included either pure Bangla text or Bangla text mixed with some characters. So secondly, the remaining comments were cleaned based on conditions like each character's Unicode value in a comment should be ranged in between 2432 to 2559 or 32 . Unicode value from 2432 to 2559 is for Bangla fonts and 32 for space. We manually labeled the dataset based on 10,000 data points. We have seven individual classes: Happy, Sad, Angry, Surprised, Excited, Abusive, and Religious.

\subsection{Data Transformation}

TF-IDF: By using TF-IDF, the dataset is transformed or encoded and features were extracted. This is a very common textto-feature vectors conversion algorithm and creates a numerical representation of a pattern used for fitting machine algorithms for predictions [21]. TF-IDF score for a word in a dataset is calculated by multiplying two different metrics [22]. The 'Term Frequency' counts how many times word $\left(\mathrm{w}_{\mathrm{i}}\right)$ appears in a document, and it is calculated by using Eq. (1).

$$
T F_{i, j}=\frac{\text { word } i \text { frequency in sentence } j}{\text { total words in sentence } j}
$$

Then the 'Inverse Document Frequency' means how common or rare a word in the entire dataset. It is calculated by using the following Eq. (2),

$$
I D F_{i}=\log \left(\frac{\text { total number of sentence }}{\text { number of sentence contain } i}\right)
$$

where IDF value of a word is the logarithm of the total number of documents or sentences divided by the number of sentences that contains a word $\left(\mathrm{w}_{\mathrm{i}}\right)$.

$$
\text { Score }_{i, j}=T F_{i, j} \times I D F_{i}
$$

where score $_{i, j}$ is the numeric value that tells how important word $\left(\mathrm{w}_{\mathrm{i}}\right)$ is. So, it can be said that the importance of a keyword phrase is determined by comparing its frequency in large sets of documents.

Let assume a small dataset: [' অাপনাকে মাঠে দেখার অপেক্ষায় রইলাম, ' আবার আপনাকে জাতীয় দলে দেখার অপেক্ষায়', ' গানের অপেক্ষা করছি কবে শুনতে পাবো]. Fig. 2 shows some sample tokens or features with their weights that are generated by TF-IDF based on the assumed dataset. TF-IDF generates tokens from every comment or sentence in a dataset, and each unique token gets a feature index. Finally, each sentence becomes a vector, and the weighted numbers of each vector represent the score of features.

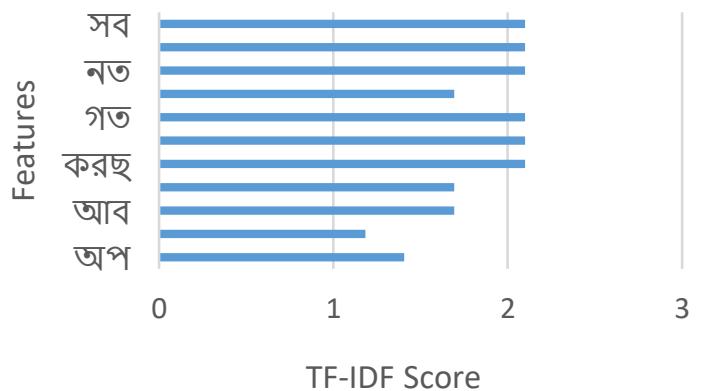

Fig. 2 Sample features with their Weights

\subsection{Proposed Classifiers}

\subsubsection{Support Vector Machine}

SVM is an algorithm that finds the best possible decision boundary between vectors that belong to the same group and those that do not belong to it. Generally, SVM works better with a small dataset, and sometimes it works better than the Neural Network-based models. This classifier categorizes ndimensional space into classes by inventing the decision boundary so that new data points can be easily placed into the appropriate category in the future [23].

By definition, SVM is a two-class model, which means it can distinguish between two different classes. To separate two classes, there can be multiple hyperplanes that can be chosen. The best hyperplane has maximum separation between two classes. Since we dealt with 7 classes, the SVM, in this case, uses the One vs. Rest (OVR) approach. It splits the multiclass dataset into multiple binary classification problems. On each binary classification problem, a binary classifier is trained, and predictions are made using the most confident model [24]. For example, our classes were Happy, Sad, Angry, Surprised, Excited, Abusive and Religious. One binary classification can be like- Happy vs [Sad, Angry, Surprised, Excited, Abusive and Religious]. So, one class is fitted against all the other classes for each classifier. It provides a significant computational advantage to the multiclass problem [9].

\subsubsection{Naïve Bayes}

Classifying using Naive Bayes is simple and relies on probabilities of events. Despite its simplicity, it performs well in various text classification problems like sentiment analysis [9]. It is a method of statistical classification that is based on the Bayes Theorem, which is as follows:

$$
P(X \mid Y)=P(X) \frac{P(Y \mid X)}{P(Y)}
$$

where,

$\mathrm{P}(\mathrm{X})=$ probability of $\mathrm{X}$ (hypothesis)

$\mathrm{P}(\mathrm{Y})=$ probability of $\mathrm{Y}($ data $)$

$\mathrm{P}(\mathrm{Y} \mid \mathrm{X})=$ porbability of $\mathrm{Y}$ given $\mathrm{X}$ that the hypothesis $\mathrm{X}$ is true $\mathrm{P}(\mathrm{X} \mid \mathrm{Y})=$ probability of $\mathrm{X}$ given $\mathrm{Y}$

We used the Multinomial NB classifier as it is suitable for this kind of research. Normally, integer feature counts are needed for the multinomial distribution. Fractional counts, such as TFIDF, can also function in practice [25]. 


\subsubsection{Neural Network}

From the three classes of Artificial Neural Network, we used Multilayer Perceptron (MLP) classifier, which is a feedforward neural network. MLPs are very flexible and usefull for tabular datasets, classification problems, regression problems, etc. In MLP, nodes are arranged in multiple layers, such as input layers, hidden layers, and output layers. MLP uses a supervised learning method called backpropagation to train its models. A linear perceptron has only one layer, but an MLP has multiple layers and non-linear activation. Data that is not linearly separable can be differentiated using this method [26].

\subsubsection{Random Forest}

The Random forest algorithm generates decision trees based on data samples and then predicts each one and selects the most appropriate solution by voting. It's an ensemble method that's better than a single decision tree because it averages the results to minimize over-fitting [27]. Fig. 3 shows the working flow of the Random Forest classifier.

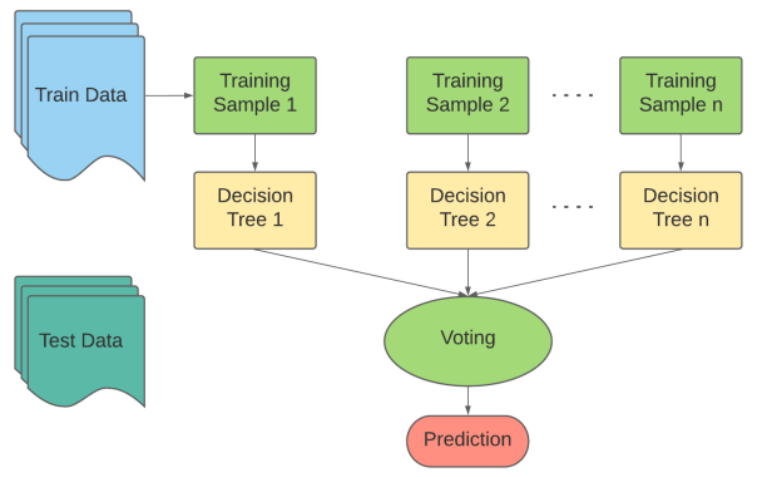

Fig. 3 Working flow of Random Forest

\subsubsection{K-Nearest Neighbors}

The KNN algorithm classifies data by identifying the $\mathrm{K}$ nearest matches in training data and then predicting using the class label of the nearest matches. Traditionally, euclidean distance has been used to find the nearest match. To calculate distance and make predictions, KNN needs a numerical representation of words that can be obtained from TF-IDF. The distance between all of these feature vectors generated by TFIDF in the data-set will be determined from the feature vector of unlabeled text data. K-Nearest vectors will be chosen from among them, and the class with the highest frequency will be labeled to the unlabeled results.

\section{Results \& Analysis}

\subsection{Results}

To better represent the proposed classifiers, we observed four different metrics called Precision, Recall, F1-Score, and Accuracy to evaluate and understand our model's performance more clearly. In every metric, 4 classifiers along with SVM were performed to observe which one works better.

\subsubsection{Precision}

The precision metric indicates how accurate the class is. This metric measures whether the prediction of the positive class is valid or not [9]. If the classifier correctly classifies all positive values, the maximum score is 1 . However, precision can be calculated by Eq. (5).

$$
\text { Precision }=\frac{T P}{T P+F P}
$$

Which is the ratio of correctly predicted positive observations to the total number of positively predicted observations. So, a more precision score means less false positive rate.

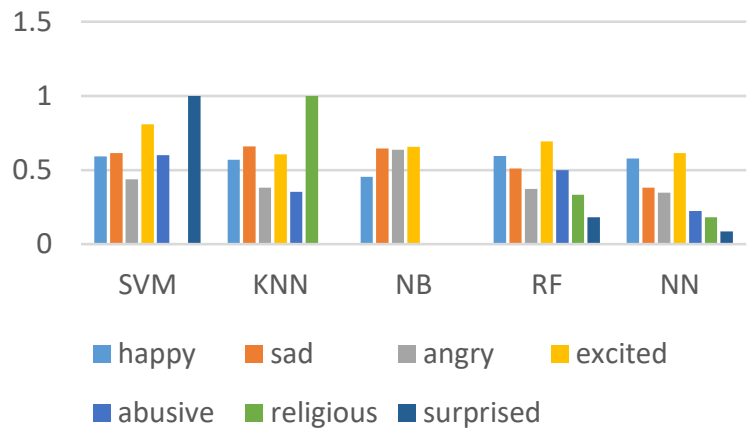

Fig. 4 Precision Score for 5 Classifiers

Fig. 4 shows that SVM has overall high Precision for all 7 classes. But it couldn't predict correctly the 'religious' class, which is here with zero precision. On the other hand, RF and NN classifiers had overall less precision value than SVM, but they could classify all of the classes. Precision alone does not help much due to its lack of consideration for the negative class. It is typically paired with the Recall metric.

\subsubsection{Recall}

The ratio of correctly expected positive observations to all observations in the actual class is known as recall [9]. Recall can be calculated by Eq. (6) where FN is considered, unlike precision.

Recall $=\frac{T P}{T P+F N}$

This metric indicates how well the model recognizes positive classes. So, a higher recall score means a lower falsepositive rate.

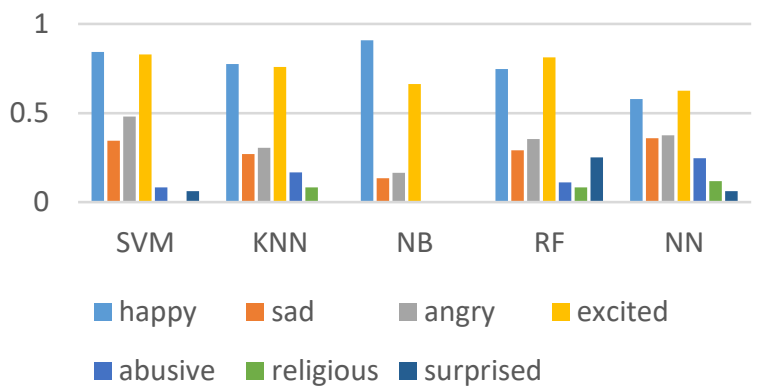

Fig. 5 Recall Score for 5 Classifiers

Fig. 5 shows that still SVM has an overall higher recall score than other classifiers but could not predict all classes correctly, as we discussed in the precision section. Since 'happy' and 'excited' classes are the majority, all of the classifiers have higher recall scores for them and a lower score for the rest of the classes. 


\subsubsection{F1 Score}

The weighted average of Precision and Recall is the F1 Score. Thus, this score takes into account both false positives and false negatives. Although $\mathrm{f} 1$ is less intuitive than accuracy, it is generally more useful if the distribution of classes is uneven [28],[29]. When false positives and false negatives cost the same, accuracy works better. If they don't, then Precision and Recall can help that's why F1 Score came. F1 score can be calculated by Eq. (7), where FN is considered, unlike precision.

$$
F 1 \text { score }=\frac{2 \times(\text { Precision } \times \text { Recall })}{\text { Precision }+ \text { Recall }}
$$

To calculate F1 Score, we used a weighted average as our dataset was imbalanced, and we wanted to give more importance to some predictions based on their proportion. It takes a weighted mean of the measures. Each weight represents the number of samples in that class. Still, we cannot say directly which classifier is better because a higher F1-score does not necessarily mean a better classifier. It combines both Precision and Recall and measures overall model accuracy. So, a high f1 score also means high Precision and Recall.

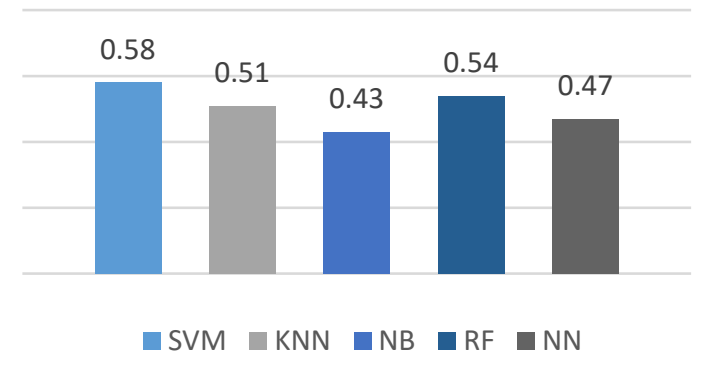

Fig. 6 Weighted F1-Scores for 5 classifiers

As shown in Fig. 6, F1 score 0.58, 0.51, 0.43, 0.54, 0.47 for SVM, KNN, NB, RF, NN respectively. SVM has a higher score than others, which means it predicted $58 \%$ of data correctly, considering both false positive and false negative values.

\subsubsection{Accuracy}

The ratio of correctly predicted True Positives and True Negatives to the total test dataset is known as accuracy. It can be calculated by Eq. (8), meaning that the number of correctly classified samples out of the total samples.

$$
\text { Accuracy }=\frac{T P+T N}{T P+T N+F P+F N}
$$

Two separate experiments were carried out to assess the accuracy of the proposed methods. We converted the dataset into two segments as $70 \%$ data for training and $30 \%$ data for testing to conduct these experiments. In the first experiment, we trained the dataset in all of the mentioned algorithms. In the second experiment, we mapped our 7 classes (Sad, Happy, Angry, Excited, Religious, Abusive, Surprised) into three higher classesPositive, Negative, and Neutral.

Table 2 Mapping 7 classes into 3 higher class

\begin{tabular}{|c|c|}
\hline New Emotion Class & Previous Emotion Class \\
\hline Positive & Happy, Excited \\
\hline Negative & Angry, Religious, Abusive \\
\hline Neutral & Surprised, Sad \\
\hline
\end{tabular}

As shown in Table 2, the mapping is done based on the 7 types of class that express actual human sentiment or thoughts. Surprised and Sad comments imply neither positive nor negative, so these were mapped into neutral class. That means the dataset is labeled with these 3 classes rather than seven. Again all of the algorithms were trained with this dataset [16].

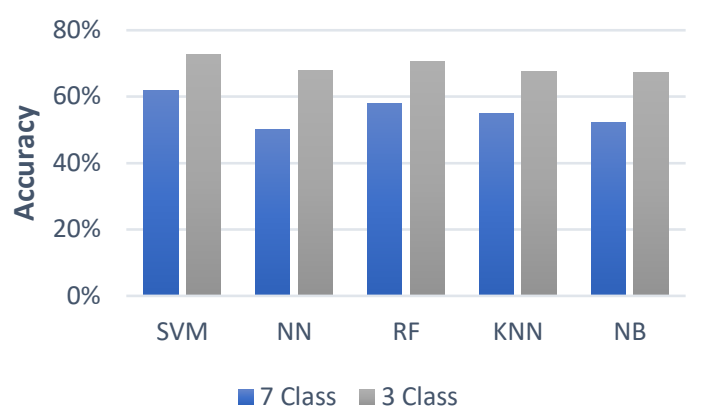

Fig. 7 Accuracy comparison between 5 classifiers

After training our algorithms with 7 class labeled dataset, we got an accuracy of $62 \%$ for Support Vector Machine (SVM), $58 \%$ for Random Forest (RF), 55\% for K-Nearest Neighbors (KNN), $52 \%$ for Naïve Bayes (NB) and $50 \%$ for Neural Network. And with 3 class labeled dataset, we got accuracy $73 \%$, $71 \%, 68 \%, 68 \%$, and $67 \%$ for SVM, RF, NN, KNN, and NB, respectively. Accuracy from two different experiments shown in Fig. 7. In both experiments, SVM worked better than the rest of the algorithms.

From the above four different types of metrics, it is cleared that SVM is the best suitable for this research as it worked better than other classifiers. This means SVM can be chosen for the future to predict human emotion towards a person or anything. Generally, this research aims to extract emotion from the comment of the different posts on social media, where people can comment on something. Many comments can be collected from social sites in an automated way, and later the proposed algorithm SVM will predict a human expression or a comment easily. For which algorithm must be trained with sample data first. So, in the future, a complete automated tool is possible to detect human sentiment.

\subsection{Validation}

Since SVM performed better in every experiment, we applied cross-validation to validate the stability and confusion matrix to evaluate performance.

\subsubsection{Confusion Matrix}

The confusion matrix is a better option for reporting results in Multi-class classification problems because it helps to observe the relations between the classifier outputs and the true ones [30]. Fig. 8 is a $7 \times 7$ Confusion matrix which is used for evaluating the performance of the SVM classifier, where 7 is the total number of target classes. The matrix compares actual target values and the ones predicted by the SVM classifier. This gives us a more comprehensive view of our classification model's performance and the errors it is making [28]. Ideally, the confusion matrix would result in values only on the diagonal. For example, the intersection cell of the $4^{\text {th }}$ row and $4^{\text {th }}$ column has a value of 0.81 which means that $81 \%$ of the 'excited' class were predicted correctly, or it can be said $81 \%$ of people are happy 
with a particular celebrity according to the test data. These values are just counts based on only test data.

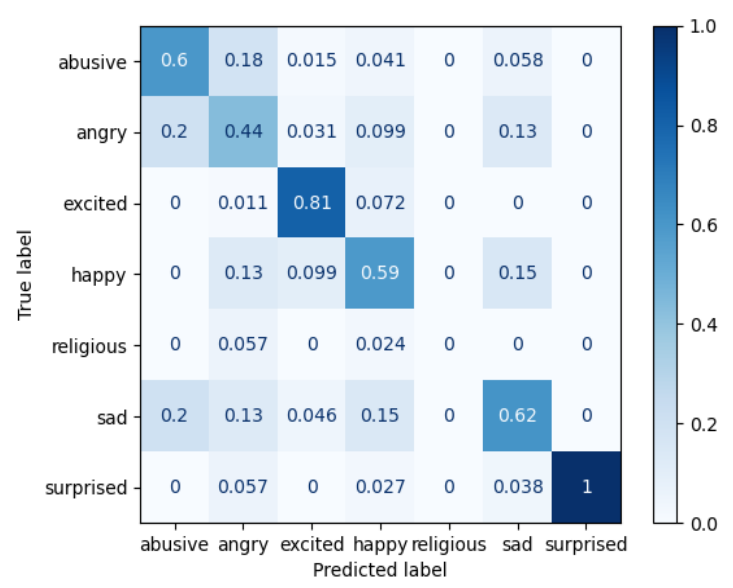

Fig. 8 Confusion Matrix for SVM

Results can be described in terms of the following:

1. True Positive (TP): Positive values were properly predicted as actual positive.

2. False Positive (FP): The predicted values were wrong in predicting a positive outcome.

3. False Negative (FN): Positive values were predicted as negative.

4. True Negative (TN): The predicted values were right in predicting a negative value.

A system is considered to be correct when it produces either true positives or true negatives. Incorrect performance occurs when the system generates False Positives or False Negatives. For example, we want to predict emotion for a sentence which is actually 'happy' type [29]. If the machine predicts emotion as 'happy' then it will be True Positive. Let's find TP, FP, FN, TN for a class say- 'abusive'.

$T P=3, T N=531, F P=2, F N=43$

Which says that 3 of 'abusive' class from test data were predicted correctly, 531 were predicted as True but not that specific class, 2 false predictions but class is true, 43 false predictions and also these were not that 'abusive' class.

\subsubsection{Cross-Validation}

It's not expected that a model will work correctly with real data it's never seen before. We need some assurance that the model has correctly identified the majority of the patterns in the dataset and is not overly sensitive to noise or has low bias and variance [29]. In order to get a static accuracy from SVM, we used cross-validation utilizing the $\mathrm{K}$-fold algorithm, which allows for random sampling, and the stratified K-fold algorithm, which allows for stratified sampling.

We got an accuracy of 58\% from K-Fold and 59\% from Stratified K-Fold cross validation (Fig. 9) for the SVM classifier. This is lower than the accuracy we've mentioned previously but it is static and much more accurate. Stratified worked well on our imbalance dataset as it does not make folds randomly.

\subsection{Limitations}

Our paper works with five different emotions and in two categories. In total, we have seven different classes and those classes are Happy, Sad, Angry, Surprised, Excited, Religious, and Abusive. By using a data collection tool we have collected almost ten thousand data. However, only three thousand data are labeled according to our classes. A major portion of our data set remains unlabeled.

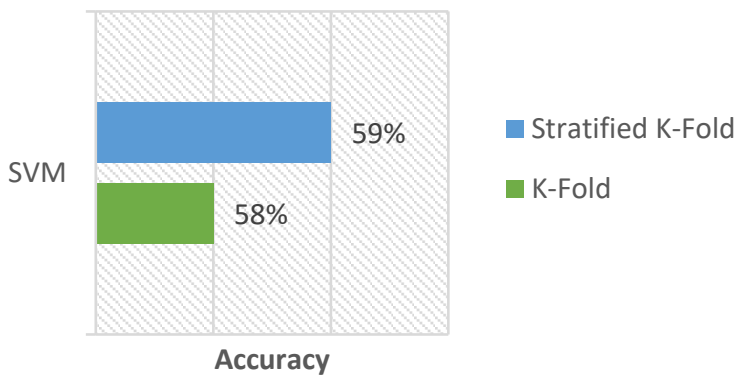

Fig. 9 Accuracy from K-Fold and Stratified K-Fold

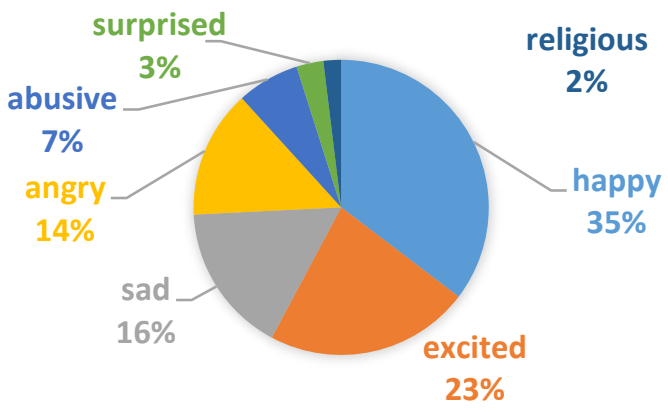

Fig. 10 Data Statistics

As a consequence, the required accuracy was not obtained according to our dataset. Due to the small dataset, Neural Network-based CNN and RNN algorithms were not used while these algorithms work well with larger datasets. Moreover, our dataset is not balanced. For seven different classes, we have different amounts of labeled data. Here, the dominating class is Happy. Almost $35 \%$ of data is labeled for the happy class, where only $2 \%$ of data is labeled for the Religious class as shown in Fig. 10. Due to a lack of balance data, we could not obtain higher accuracy.

\section{Conclusion}

Sentiment analysis is playing an essential role in revealing convenient information from people's opinions. It helps us to extract human emotions through speech, text, or even facial expressions. As a result, extracting viewpoints from text, speech, and facial expressions is a critical challenge in various fields, including social media tracking, product analysis, customer reviews analysis, clinical service analysis, and so on. Being inspired by these needs, this research paper works on extracting emotion from Bangla text. We know people nowadays are very involved in social media such and they are using social media to express their opinions on various matters. So, sentiment analysis can be helpful to gain a deeper understanding of people's views in order to provide better service. This study aimed to collect comments from different Facebook pages of Bangladeshi celebrities and develop a model that will be able to predict sentiment based on Bengali text. Our proposed methods can predict five other emotions from Bangla text, and we achieved $62 \%$ accuracy by using the Support Vector Machine (SVM) machine learning algorithm. Apart from this, our method is able to predict two different categories, such as Religious and 
Abusive, which made our research work unique from other existing research work. In addition, the main reason for choosing SVM as the primary model is that it works better than other models such as Random Forest (RF), K-Nearest Neighbors (KNN), Naïve Bayes (NB), and Neural Network that is described in the previous section.

\section{References}

[1] Panksepp J., 2004. Affective neuroscience: The foundations of human and animal emotions. Oxford university press.

[2] Damasio A.R., 1998. Emotion in the perspective of an integrated nervous system. Brain research reviews, 26(2-3), pp.83-86.

[3] Ekman. P.E. and Davidson, R.J., 1994. The nature of emotion: Fundamental questions. Oxford University Press.

[4] Viking Mäntylä., M. Graziotin, D. and Kuutila M., 2016. The Evolution of Sentiment Analysis-A Review of Research Topics, Venues, and Top Cited Papers. arXiv e-prints, pp.arXiv-1612.

[5] Drovo, M.D., Chowdhury, M. Uday, S.I. and Das. A.K., 2019, June. Named entity recognition in bengali text using merged hidden markov model and rule base approach. In 2019 7th International Conference on Smart Computing \& Communications (ICSCC) (pp. 1-5). IEEE.

[6] Hossain, M.T., Hasan, M.W. and Das, A.K., 2021, January. Bangla Handwritten Word Recognition System Using Convolutional Neural Network. In 2021 15th International Conference on Ubiquitous Information Management and Communication (IMCOM) (pp. 1-8). IEEE.

[7] Mumu, T.F., Munni, I.J. and Das, A.K., 2021. Depressed people detection from bangla social media status using lstm and cnn approach. Journal of Engineering Advancements, 2(01), pp.4147.

[8] Islam, J., Mubassira, M., Islam, M.R. and Das, A.K., 2019, February. A speech recognition system for Bengali language using recurrent neural network. In 2019 IEEE 4th international conference on computer and communication systems (ICCCS) (pp. 73-76). IEEE.

[9] Mahmudun, M., Altaf, M.T. and Ismail, S., 2016. Detecting sentiment from Bangla text using machine learning technique and feature analysis. Int. J. Comput. Appl., 975, p.8887.

[10] Das, A. and Bandyopadhyay, S., 2010. Phrase-level polarity identification for Bangla. Int. J. Comput. Linguist. Appl.(IJCLA), 1(1-2), pp.169-182.

[11] Das, D., 2011, March. Analysis and tracking of emotions in english and bengali texts: a computational approach. In Proceedings of the 20th international conference companion on World wide web (pp. 343-348).

[12] Hasan, K.A. and Rahman, M., 2014, December. Sentiment detection from bangla text using contextual valency analysis. In 2014 17th International Conference on Computer and Information Technology (ICCIT) (pp. 292-295). IEEE.

[13] Chowdhury, S. and Chowdhury, W., 2014, May. Performing sentiment analysis in Bangla microblog posts. In 2014 International Conference on Informatics, Electronics \& Vision (ICIEV) (pp. 1-6). IEEE.

[14] Go, A., Bhayani, R. and Huang, L., 2009. Twitter sentiment classification using distant supervision. CS224N project report, Stanford, 1(12), p.2009.

[15] Pandey, P. and Govilkar, S., 2015. A framework for sentiment analysis in Hindi using HSWN. International Journal of Computer Applications, 119(19).

[16] Tuhin, R.A., Paul, B.K., Nawrine, F., Akter, M. and Das, A.K., 2019, February. An automated system of sentiment analysis from bangla text using supervised learning techniques. In 2019
IEEE 4th International Conference on Computer and Communication Systems (ICCCS) (pp. 360-364). IEEE.

[17] Tembhurnikar, S.D. and Patil, N.N., 2015. Sentiment Analysis using LDA on Product Reviews: A Survey. International Journal of Computer Applications, 975, p.8887.

[18] Umamaheswari, K. and Karthiga, R., Sentiment Classification based on Latent Dirichlet Allocation. International Journal of Computer Applications, 975, p.8887.

[19] Das, A.K., Al Asif, A., Paul, A. and Hossain, M.N., 2021. Bangla hate speech detection on social media using attentionbased recurrent neural network. Journal of Intelligent Systems, 30(1), pp.578-591.

[20] Datacamp.com, 2021. [Online]. Available: https://www.datacamp.com/community/tutorials/svmclassification-scikit-learn-python.

[21] Rakib, O.F., Akter, S., Khan, M.A., Das, A.K. and Habibullah, K.M., 2019, December. Bangla word prediction and sentence completion using GRU: an extended version of RNN on N-gram language model. In 2019 International Conference on Sustainable Technologies for Industry 4.0 (STI) (pp. 1-6). IEEE.

[22] Monkeylearn.com, 2021. [Online]. Available: https://monkeylearn.com/blog/what-is-tf-idf/

[23] "Support Vector Machine (SVM) Algorithm - Javatpoint", www.javatpoint.com, 2021. [Online]. Available: https://www.javatpoint.com/machine-learning-support-vectormachine-algorithm.

[24] Hossain, M.M., Labib, M.F., Rifat, A.S., Das, A.K. and Mukta, M., 2019, June. Auto-correction of english to bengali transliteration system using levenshtein distance. In 2019 7th International Conference on Smart Computing \& Communications (ICSCC) (pp. 1-5). IEEE.

[25] Labib, M.F., Rifat, A.S., Hossain, M.M., Das, A.K. and Nawrine, F., 2019, June. Road accident analysis and prediction of accident severity by using machine learning in Bangladesh. In 2019 7th International Conference on Smart Computing \& Communications (ICSCC) (pp. 1-5). IEEE.

[26] "Multilayer Perceptron (MLP) vs Convolutional Neural Network in Deep Learning", Medium, 2021. [Online]. Available: https://medium.com/data-sciencebootcamp/multilayer-perceptron-mlp-vs-convolutional-neuralnetwork-in-deep-learning-c890f487a8f1.

[27] Emon, E.A., Rahman, S., Banarjee, J., Das, A.K. and Mittra, T., 2019, June. A deep learning approach to detect abusive bengali text. In 2019 7th International Conference on Smart Computing $\&$ Communications (ICSCC) (pp. 1-5). IEEE.

[28] Ullah, M.R., Bhuiyan, M.A.R. and Das, A.K., 2017, September. IHEMHA: Interactive healthcare system design with emotion computing and medical history analysis. In 2017 6th International Conference on Informatics, Electronics and Vision \& 2017 7th International Symposium in Computational Medical and Health Technology (ICIEV-ISCMHT) (pp. 1-8). IEEE.

[29] Bhuiyan, M., Rahman, A., Ullah, M. and Das, A.K., 2019. iHealthcare: Predictive model analysis concerning big data applications for interactive healthcare systems. Applied Sciences, 9(16), p.3365.

[30] "Confusion Matrix - an overview | ScienceDirect Topics", Sciencedirect.com, 2021. [Online]. Available: https://www.sciencedirect.com/topics/engineering/confusionmatrix.

[31] Das, A.K., Ashrafi, A. and Ahmmad, M., 2019, February. Joint cognition of both human and machine for predicting criminal punishment in judicial system. In 2019 IEEE 4th International Conference on Computer and Communication Systems (ICCCS) (pp. 36-40). IEEE. 